\title{
Étude préliminaire des communautés ichtyofauniques de la lagune Ono (Côte d'Ivoire)
}

\author{
Annigbé Justin EYI ${ }^{1 *}$, Kouadio Justin KONAN², Kouassi TANO ${ }^{3}$, Konan N'DA ${ }^{1}$, Boua Celestin ATSE 2 \\ 1 Université Nangui Abrogoua, ${ }^{2}$ Centre de Recherches Océanologiques, ${ }^{3}$ Université Félix Houphouët Boigny. \\ Auteur correspondant ${ }^{\text {* }}$ \\ ${ }_{1}^{1 *} E Y I$ Annigbé Justin, Université Nangui Abrogoua, 02 BP 801 ABIDJAN 02, Côte d'Ivoire \\ E-mail : justineyi@yahoo.fr, Téléphone : +225 07417150
}

Original submitted in on $9^{\text {th }}$ June 2016. Published online at www.m.elewa.org on $31^{\text {st }}$ August 2016

http://dx.doi.org/10.4314/jab.v104i1.1

\section{RESUME}

Objectifs : Le présent travail vise à étudier la structure et l'organisation des peuplements ichtyologiques de la lagune Ono.

Méthodologie et Résultats : Les poissons ont été échantillonnés mensuellement de septembre 2015 à février 2016 à l'aide de nasses, de filets maillants, d'harpons et d'éperviers. Au total, 39 espèces appartenant à 28 genres, 23 familles et 8 ordres ont été capturées. Les captures sont dominées en termes d'abondance et de biomasse par les espèces dulçaquicoles $(n=31)$ représentant $79,49 \%$ contre 06 espèces à affinité marine et/ou estuarienne $(15,38 \%)$ et 02 espèces introduites $(5,12 \%)$. Les indices de diversité spécifique et d'équitabilité enregistrés sont respectivement de 4,44 bits/individus et 0,85 . Les ordres renfermant le plus grand nombre de familles et d'espèces sont les Perciformes ( 9 familles et 18 espèces) suivi des Ostéoglossiformes et des Characiformes (respectivement 3 et 5 espèces chacune). Les Cypriniformes sont représentés par une seule famille (Cyprinidae) et trois espèces tandis que les Mugiliformes, les Clupéiformes et les Eloptiformes sont représentés par une seule espèce.

Conclusion et application : Cette étude a permis de connaitre la communauté ichtyologique de la lagune Ono. Face à l'eutrophisation croissante de cette lagune, ces résultats serviront de références pour les investigations ultérieures de suivi de l'état de la faune ichtyologique et à l'élaboration de mesures adéquates pour éviter la perte de la biodiversité aquatique.

Mots clés : Distribution, ichtyofaune, Eutrophisation, lagune Ono, structure, Côte d'Ivoire.

\section{Preliminary study of ichtyofaunic communities of the Ono lagoon (Ivory Coast)}

\begin{abstract}
Objectives: The present work aims to study the structure and organization of the fish communities of the Ono lagoon.

Methodology and Results: Fish were sampled monthly from September 2015 to February 2016 using traps, gill nets, harpoons and hawks. A total of 39 species belonging to 28 genera, 23 families and 8 orders was captured. Catches are dominated in term of abundance and biomass by freshwater species $(n=31)$ representing $79.49 \%$ against 06 marine and/or estuarine species $(20.51 \%)$ and 02 introduced species
\end{abstract}


(5.12\%).Specific diversity and equitability indices recorded were 4.44 bits / individuals and 0.85 respectively. The orders including the highest number of families and species were Perciformes ( 9 families and 18 species) followed by Osteoglossiformes and Characiformes ( 3 and 5 species respectively). Cypriniformes were represented by a single family (Cyprinidae) and three species whilst Mugiliformes, Clupeiformes and Eloptiformes were represented by only one species.

Conclusion and application: This study allowed to know the fish community of Ono lagoon. Given the increasing eutrophication of the lagoon, these results will be a reference for further investigations of monitoring the state of the fish fauna and the development of adequate measures to prevent the loss of aquatic biodiversity.

Keywords : Distribution, ichthyofauna, Eutrophication Ono lagoon, structure, Ivory Coast.

\section{INTRODUCTION}

Les écosystèmes aquatiques fournissent beaucoup de biens et de services qui ne sont pas souvent appréciés à leur juste valeur (Brummett et al., 2008). En Afrique la connaissance de la faune l'ichtyologique a suscité depuis fort longtemps l'intérêt des scientifiques. C'est d'ailleurs l'un des rares continents pour lesquels nous disposons d'un inventaire des espèces d'eau douce et saumâtre (Lévêque, 1994). Dans le cadre général de la région ouest-africaine, la faune ichtyologique de la Côte d'lvoire a été étudiée de façon approfondie (Teugels et al., 1988 ; Gourène et al., 1999). La région du sud-Comoé qui abrite les complexes lagunaires Ebrié/Aby et le fleuve Comoé a été largement étudiée (Daget et Iltis, 1965 ; Teugels et al., 1988 ; Albaret, 1994 ; Guiral et al., 1999). La lagune Ono, d'une superficie d'environ 400 ha, est un est petit écosystème situé à l'extrême Est du complexe lagunaire Ebrié. Sa proximité avec les unités agro-industrielles de l'ex Société Alsacienne de Côte d'Ivoire (SALCI) et de la Société de l'étude pour la Culture Bananière (SCB) et des eaux de lessivage des terres agricoles avoisinantes sont source permanente de

\section{MATERIEL ET METHODES}

Milieu d'étude : La lagune Ono est une lagune côtière d'eau douce située entre les longitudes $3^{\circ} 33^{\prime} 53^{\prime \prime}$ Ouest et les latitudes $5^{\circ} 22^{\prime} 22^{\prime}$ 'Nord à 12 kilomètres de la ville de Bonoua, dans le département de GrandBassam. Elle a une superficie d'environ 400 ha et s'ouvre sur le fleuve Comoé qui se jette à son tour dans l'océan Atlantique. Cette unité fonctionne comme un réservoir d'eau douce car alimentée essentiellement par la nappe phréatique et non comme une véritable lagune. Selon Guiral et Ferhi (1989), la composition pollution. En effet, le bassin versant de cette lagune renferme d'importantes zones d'occupation humaine et des grandes plantations industrielles et villageoises de cultures pérennes. Les activités anthropiques qui y sont menées sont susceptibles d'influencer négativement non seulement la qualité écologique mais aussi les communautés biologiques en général et particulièrement l'ichtyofaune. En outre, l'accentuation de l'envahissement par les plantes aquatiques et surtout par Hydrilla verticillata induit un enrichissement en matières en suspension, source de dégradation de la qualité de l'eau. La nécessité de prendre des mesures de conservation devient dès lors une préoccupation majeure puisque ces mesures pour être efficaces nécessitent une bonne connaissance des espèces et des relations qui les lient à leur milieu. En dehors des connaissances sur les macrophytes de cette lagune (Ettien et Guiral, 1991), aucune étude relative à la faune ichtyologique n'a été abordée jusqu'à ce jour. Le présent travail se propose d'étudier la structure et l'organisation des peuplements ichtyologiques de la lagune Ono.

chimique et la teneur en radio-isotope de l'oxygène 18 $\left({ }^{18} \mathrm{O}\right)$ diffère en particuliers des valeurs enregistrées dans le système lagunaire Ebrié. Cette lagune est envahie par plusieurs macrophytes flottantes, réduisant de façon considérable la surface exploitable de l'eau. II s'agit entre autres, de Echinochloa pyramidalis, de Eichhornia crassipes, de Salvinia molesta et ces cinq dernières années de Hydrilla verticillata.

Échantillonnage des poissons : Les échantillonnages ont été effectués au niveau des sites de débarquement 


\section{Eyi et al. J. Appl. Biosci. 2016 Étude préliminaire des communautés ichtyofauniques de la lagune Ono}

(Côte d'Ivoire).

de la pêche artisanale de septembre 2015 et février 2016. Les captures proviennent d'une panoplie d'engins de pêche (nasses, filets maillants, harpons et éperviers) opérant sur tout l'ensemble de la surface exploitable de la lagune Ono (Figure 1). Les espèces rencontrées ont été systématiquement photographiées puis identifiées à l'aide des clés d'identification de Teugels et al. (1988) et de Paugy et al. (2003). Ces espèces ont été ensuite classées en grandes catégories écologiques selon Lévêque et Paugy et al., 2006. Après échantillonnage, chaque spécimen a été mesuré au millimètre près puis pesé au centième de gramme près.

Mesure des paramètres physico-chimiques: Les paramètres physico-chimiques ont été relevés pendant toute la période d'étude à l'aide d'un multi paramètre de type HANNA. II s'agit entre autres, de la température, de la salinité, de l'oxygène dissous, du $\mathrm{pH}$, du taux de solides dissous et de la conductivité. Ces mesures ont été effectuées in situ en amont en aval et au centre de la lagune à une profondeur d'au moins $50 \mathrm{~cm}$.

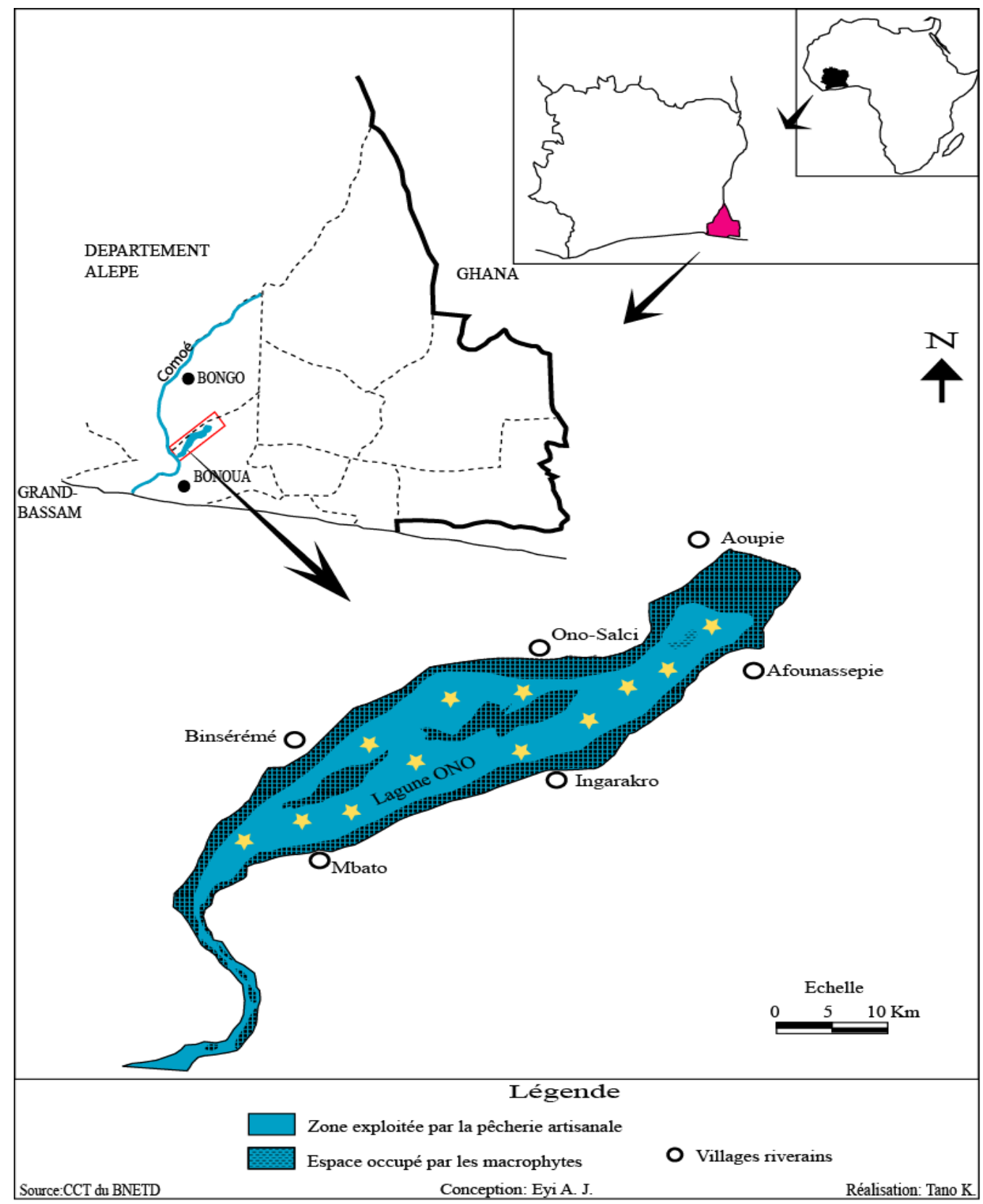


Figure 1 : Carte de localisation de la lagune Ono et des zones exploitées ( ) sar la pêcherie artisanale

Traitement des données : Les données collectées ont servi à déterminer la richesse spécifique, l'indice de diversification des espèces au sein des familles, l'indice de diversité spécifique de Shannon et l'indice d'équitabilité de Pielou afin de caractériser le degré et la qualité de l'organisation du peuplement. L'indice de diversité spécifique mesure la répartition de l'abondance (effectif ou biomasse) entre les taxa. L'indice de diversité spécifique de Shannon $\left(\mathrm{H}^{\prime}\right)$ varie de 0 pour une seule espèce à $\log _{2}(S)$ lorsque toutes les espèces ont la même abondance. Sa formule est la suivante :

$$
\mathrm{H}^{\prime}=-\sum_{\mathrm{i}=1}^{\mathrm{S}} \mathrm{P}_{\mathrm{i}}=\log _{2}\left(\mathrm{P}_{\mathrm{i}}\right)
$$

Avec $P_{i}=$ abondance proportionnelle de l'espèce $\left(P_{i}=\right.$ $\left.n_{i} / N\right)$,

$n_{i}=$ Nombre d'individus d'une espèce dans l'échantillon, $\mathrm{N}=$ nombre total d'individus de toutes les espèces dans l'échantillon.

L'indice d'équitabilité ( $E$ ) a été calculé selon Pielou (1975) par la formule :

$\mathrm{E}=\mathrm{H}^{\prime} / \log _{2}(\mathrm{~S})$

Avec $H^{\prime}=$ diversité spécifique et $\log _{2}(S)=$ diversité maximale et $S=$ nombre des espèces dans l'échantillon.

\section{RESULTATS}

Paramètres physico-chimiques : Les paramètres physicochimiques de l'eau ont varié significativement $(p<0,05)$ à l'exception de la salinité (Tableau 1).

Tableau 1 : valeurs moyennes des paramètres physico-chimiques enregistrées en lagune Ono de septembre 2015 à février 2016.

\begin{tabular}{l|c|c|c|c|c|c}
\hline Paramètres & Septembre & Octobre & Novembre & Décembre & Janvier & Février \\
\hline Température $\left({ }^{\circ} \mathrm{C}\right)$ & $25,93 \pm 0,98^{\mathrm{a}}$ & $28,73 \pm 0,84^{\mathrm{b}}$ & $26,48 \pm 1,05^{\mathrm{a}}$ & $25,72 \pm 0,06^{\mathrm{a}}$ & $27,02 \pm 1,03^{\mathrm{ab}}$ & $29,32 \pm 0,91^{\mathrm{b}}$ \\
\hline $\mathrm{pH}$ & $5,64 \pm 0,09^{\mathrm{ab}}$ & $6,34 \pm 0,58^{\mathrm{b}}$ & $5,87 \pm 0,12^{\mathrm{ab}}$ & $5,39 \pm 0,38^{\mathrm{a}}$ & $5,43 \pm 0,03^{\mathrm{a}}$ & $5,72 \pm 0,24^{\mathrm{ab}}$ \\
\hline Oxygène dissous $(\mathrm{mg} / \mathrm{L})$ & $1,33 \pm 1,10^{\mathrm{a}}$ & $5,42 \pm 0,38^{\mathrm{b}}$ & $1,08 \pm 0,73^{\mathrm{a}}$ & $1,87 \pm 0,22^{\mathrm{a}}$ & $1,25 \pm 0,77^{\mathrm{a}}$ & $2,20 \pm 1,12^{\mathrm{a}}$ \\
\hline Salinité $(\mathrm{PSU})$ & $0,01 \pm 0.00$ & $0,01 \pm 0,00$ & $0,01 \pm 0,00$ & $0,01 \pm 0,01$ & $0,01 \pm 0,00$ & $0,01 \pm 0,00$ \\
\hline Conductivité $(\mu \mathrm{s} / \mathrm{cm})$ & $21,22 \pm 1,67^{\mathrm{ab}}$ & $20,55 \pm 2,99^{\mathrm{a}}$ & $22,78 \pm 0,69^{\mathrm{b}}$ & $24,00 \pm 1,73^{\mathrm{b}}$ & $25,22 \pm 1,68^{\mathrm{b}}$ & $17,66 \pm 1,00^{\mathrm{a}}$ \\
\hline $\begin{array}{l}\text { Taux de Solides Dissous } \\
(\mathrm{mg} / \mathrm{L})\end{array}$ & $10,77 \pm 0,69^{\mathrm{ab}}$ & $10,22 \pm 1,27^{\mathrm{a}}$ & $11,44 \pm 0,38^{\mathrm{a}}$ & $13,67 \pm 0,58^{\mathrm{b}}$ & $13,11 \pm 1,95^{\mathrm{b}}$ & $8,67 \pm 0,58^{\mathrm{a}}$ \\
\hline
\end{tabular}

Les valeurs de la température obtenues sont comprises entre $25,72{ }^{\circ} \mathrm{C}$ et $29,32{ }^{\circ} \mathrm{C}$ avec des valeurs relativement faibles en décembre et élevées en février. Les valeurs du $\mathrm{pH}$, de l'oxygène dissous et de la salinité ont été faibles tout le long de la période d'étude.
Le pourcentage d'occurrence ou de fréquence renseigne sur les préférences de milieu (habitat) d'une espèce donnée. Elle consiste à compter le nombre de fois où l'espèce «il» apparaît dans les captures (Dajoz, 2000). II se calcule comme suit :

$\mathrm{F}=\frac{\mathrm{F}_{\mathrm{i}}}{\mathrm{F}_{\mathrm{t}}} \times 100$

Avec $F_{i}=$ nombre de débarquements contenant l'espèce $\mathrm{i}$ et $F_{t}=$ nombre total de débarquements effectués.

En fonction de la valeur de $F$, trois classes d'occurrence ont été déterminées. II s'agit des espèces constantes ( $F>50 \%)$, des espèces accessoires (25< $F$ $<50 \%$ ) et des espèces accidentelles ( $F<25 \%$ ).

Analyses statistiques : Les variations mensuelles des paramètres physico-chimiques ont été comparées à l'aide d'ANOVA à un facteur. Les différences sont considérées significatives à $p<0,05$. Le test de Tukey a été utilisé pour les comparaisons a posteriori. Les variations saisonnières des indices de Shannon $\left(H^{\prime}\right)$ et de Piélou (E) ont été comparées au moyen du test de Mann-Whitney : $p>0,05$. Les analyses ont été effectuées avec le logiciel STATISTICA 7.1 (Statsoft inc.).
Les valeurs obtenues oscillent entre $5,39 \pm 0,38$ et $6,34 \pm 0,58$ pour le $\mathrm{pH}$ entre $1,08 \pm 0,73 \mathrm{mg} / \mathrm{L}$ et $5,42 \pm 0,38 \mathrm{mg} / \mathrm{L}$ pour l'oxygène dissous et autour de 0,01 PSU pour la salinité. Pour la conductivité, les plus fortes valeurs sont observées en janvier $(13,11 \pm 1,95$ 

(Côte d'Ivoire).

$\mu s / \mathrm{cm})$ tandis que les plus faibles valeurs sont enregistrées en février $(8,67 \pm 0,58 \mu \mathrm{s} / \mathrm{cm})$. Celles relatives au Taux de Solides Dissous sont élevées en décembre $(13,67 \pm 0,58 \mathrm{mg} / \mathrm{L})$ et plus faible en février $(8,67 \pm 0,580 \mathrm{mg} / \mathrm{L})$.

Communautés ichtyologiques : Au total, 39 espèces appartenant à 28 genres, 23 familles et 8 ordres ont été échantillonnées au cours de cette étude (Tableau 2). Les ordres renfermant le plus grand nombre de familles et d'espèces sont les Perciformes ( 9 familles et 18 espèces), suivis des Ostéoglossiformes et des Characiformes (respectivement 3 et 5 espèces chacune). Les Cypriniformes sont représentés par une seule famille (Cyprinidae) et trois espèces tandis que les Mugiliformes, les Clupéiformes et les Eloptiformes sont représentés chacun par une seule famille et une seule espèce. La famille la plus représentée est celle des Cichlidae avec 10 espèces, soit 25,64\% des individus capturés. Elle est suivie des Mormyridae et des Alestidae qui regroupent chacune 3 espèces soit $7,69 \%$ des proportions puis des Claroteidae et des Cyprinidae qui renferment chacune 2 espèces $(5,13$ $\%)$. Les autres familles ne sont représentées que par une seule espèce chacune. En termes de nombre d'individus échantillonnés, les familles dominantes sont les Cichlidae avec $45 \%$ des effectifs suivies des Claroteidae $24 \%$ et des Mormyridae $6 \%$. Par ailleurs, 31 espèces à affinité continentale représentant 79,49\% des captures contre 06 espèces à affinité marine et/ou estuarienne $(15,38 \%)$ et 02 espèces introduites $(5,13$ $\%)$ ont été enregistrées. Les espèces à affinité marine et/ou estuarienne sont composées de Liza falcipinnis, de Pellonula leonensis, de Elops lacerta, de Sarotherodon melanotheron, de Tilapia guineensis, de Tylochromis jentinki, de Pomadasys jubelini et de Sphyraena afra. En fonction des taux d'occurrence, les différentes espèces pêchées dans la lagune Ono ont été classées en 8 espèces constantes (Chrysichthys nigrodigitatus, Parachanna obscura, Papyrocranus afer, Mormyrops anguilloides, Sarotherodon melanotheron, Hemichromis fasciatus, Hemichromis bimaculatus, et le Tilapia hybride (Tilapia guineensis $x$ Tilapia zillii) et 8 espèces accessoires (Oreochromis niloticus, Tilapia guineensis, Tilapia zillii, Tylochromis jentinki, Marcusenius furcidens, Marcusenius ussheri, Tilapia busumana et Tilapia mariae). Les 23 autres espèces de la liste ont été considérées comme accidentelles.

Tableau 2 : Composition des communautés ichtyologiques rencontrées en lagune Ono.

\begin{tabular}{l|l|c|c|c}
\hline \multicolumn{1}{c}{ Taxons } & $\begin{array}{l}\text { Abond } \\
\text { ance }\end{array}$ & $\%$ F & Classe d'occurrence \\
\hline SILURIFORMES & \multicolumn{1}{c|}{} & & & \\
\hline CLARIIDAE & Clarias ebriensis Pellegrin, 1920 & + & 14 & Accidentelle \\
\hline CLAROTEIDAE & Chrysichthys maurus (Valenciennes, 1840) & + & 23 & Accidentelle \\
\hline & Chrysichthys nigrogitatus (Lacépède, 1803) & +++ & 100 & Constante \\
\hline SCHILBEIDAE & Schilbe mandibularis (Günther, 1867) & + & 18 & Accidentelle \\
\hline MALAPTERURIDAE & Malapterurus electricus (Gmelin, 1789) & + & 6 & Accidentelle \\
\hline MUGILIFORMES & & & & \\
\hline MUGILIDAE & Liza falcipinnis (Valencienne, 1836) & \\
\hline CHARACIFORMES & & + & 16 & Accidentelle \\
\hline HEPSETIDAE & Hepsetus odoe (Bloch, 1794) & & & \\
\hline ALESTIDAE & Brycinus imberi (Peters, 1852) & + & 14 & Accidentelle \\
\hline & Brycinus macrolepidotus Valenciennes, 1849 & + & 15 & Accidentelle \\
\hline DISTICHODONTIDAEE & Brycinus nurse (Rüppel, 1832) & + & 14 & Accidentelle \\
\hline Cistichodus rostratus Günther, 1864 & + & 14 & Accidentelle \\
\hline CYPRINIFORMES & & + & 09 & Accidentelle \\
\hline CYPRINIDAE & Barbus ablabes (Bleeker, 1863) & & & \\
\hline & Barbus trispilis (Bleeker, 1863) & + & 12 & Accidentelle \\
\hline CLUPEIFORMES & Labeo courbie (Rüppel, 1832) & + & 10 & Accidentelle \\
\hline CLUPEIDAE & Pellonula leonensis Boulenger, 1916* & + & 18 & Accidentelle \\
\hline ELOPTIFORMES & & + & 11 & Accidentelle \\
\hline
\end{tabular}


Eyi et al. J. Appl. Biosci. 2016 Étude préliminaire des communautés ichtyofauniques de la lagune Ono (Côte d'Ivoire).

\begin{tabular}{|c|c|c|c|c|}
\hline ELOPTIDAE & Elops lacerta Valenciennes, $1846^{*}$ & + & 12 & Accidentelle \\
\hline \multicolumn{5}{|c|}{ Tableau 2 (suite et fin) } \\
\hline \multicolumn{5}{|l|}{ PERCIFORMES } \\
\hline CHANNIDAE & Parachanna obscura (Günther, 1861) & ++ & 85 & Constante \\
\hline \multirow[t]{10}{*}{ CICHLIDAE } & Hemichromis bimaculatus Gill, 1862 & ++ & 67 & Constante \\
\hline & Hemichromis fasciatus Peters, 1852 & ++ & 73 & constante \\
\hline & Oreochromis niloticus (Linnaeus, 1758) ** & ++ & 45 & Accessoire \\
\hline & Sarotherodon melanotheron Rüppell, $1852^{*}$ & +++ & 100 & Constante \\
\hline & Tilapia busumana (Günther, 1903) & +++ & 34 & Accessoire \\
\hline & Tilapia guineensis (Bleeker, 1862)* & ++ & 33 & Accessoire \\
\hline & Tilapia zillii (Gervais, 1848) & ++ & 26 & Accessoire \\
\hline & Tylochromis jentinki (Steindachner, 1895)* $^{*}$ & ++ & 47 & Accessoire \\
\hline & Tilapia mariae (Vervoort, 1980) & ++ & 43 & Accessoire \\
\hline & Hybride Tilapia (T. guineensis× Tilapia zillii) & +++ & 100 & Constante \\
\hline GOBIIDAE & Sicyopterus lagocephalus (Pallas, 1770) & + & 23 & Accidentelle \\
\hline POLYNEMIDAE & Galeoides decadactylus (Bloch, 1795) & + & 7 & Accidentelle \\
\hline ANABANTIDAE & Ctenopoma petherici (Günther, 1864) & ++ & 24 & Accidentelle \\
\hline HAEMULIDAE & Pomadasys jubelini (Lacépède, 1802)* & + & 12 & Accidentelle \\
\hline SPHYRAENIDAE & Sphyraena afra (Peters, 1844) & + & 6 & Accidentelle \\
\hline POLIMIDAE & Polydactylus quadrifilis (Cuvier, 1829) & + & 8 & Accidentelle \\
\hline MONODACTYLIDAE & Monodactylus sebae (Cuvier, 1829) & + & 6 & Accidentelle \\
\hline \multicolumn{5}{|c|}{ OSTEOGLOSSIFORMES } \\
\hline NOTOPTERIDAE & Papyrocranus afer (Günther, 1868) & ++ & 78 & Constante \\
\hline \multirow[t]{3}{*}{ MORMYRIDAE } & Marcusenius furcidens (Pellegrin, 1920) & ++ & 47 & Accessoire \\
\hline & Marcusenius ussheri (Günther, 1867) & + & 49 & Accessoire \\
\hline & Mormyrops anguilloides (Linnaeus, 1758) & ++ & 55 & Constante \\
\hline OSTEOGLOSSIDAE & Heterotis niloticus (Cuvier, 1829) ** & + & 22 & Accidentelle \\
\hline TOTAL & 39 & & & \\
\hline
\end{tabular}

$1^{+}=$rare $;++=$abondant $;+++=$très abondant $;{ }^{*}=$ espèces à affinité marine et /ou estuarienne (Lévêque et Paugy et al., 2006); ** = espèces introduites dans les hydrosystèmes de la Côte d'ivoire ;

$\% \mathrm{~F}=$ pourcentage d'occurrence).

Composition temporelle du peuplement: La composition des captures durant la saison des pluies en termes d'individus est dominée par les Cichlidae $(31 \%)$, suivis des Claroteidae $(25 \%)$, des Mormyridae $(8 \%)$ et des Alestidae (6\%), (Figure $2 \mathrm{~A})$. En saison sèche, le même ordre a été respecté avec une légère augmentation du taux des Cichlidae $(37 \%)$, des Claroteidae (29\%). Les Mormyridae et les Alestidae ont eu leur pourcentage inchangé. Aucune espèce de la famille des Sphyraenidae, des Polymidae et des Haemulidae n'a été capturée pendant cette saison (Figure 2B). 
Eyi et al. J. Appl. Biosci. 2016 Étude préliminaire des communautés ichtyofauniques de la lagune Ono (Côte d'Ivoire).
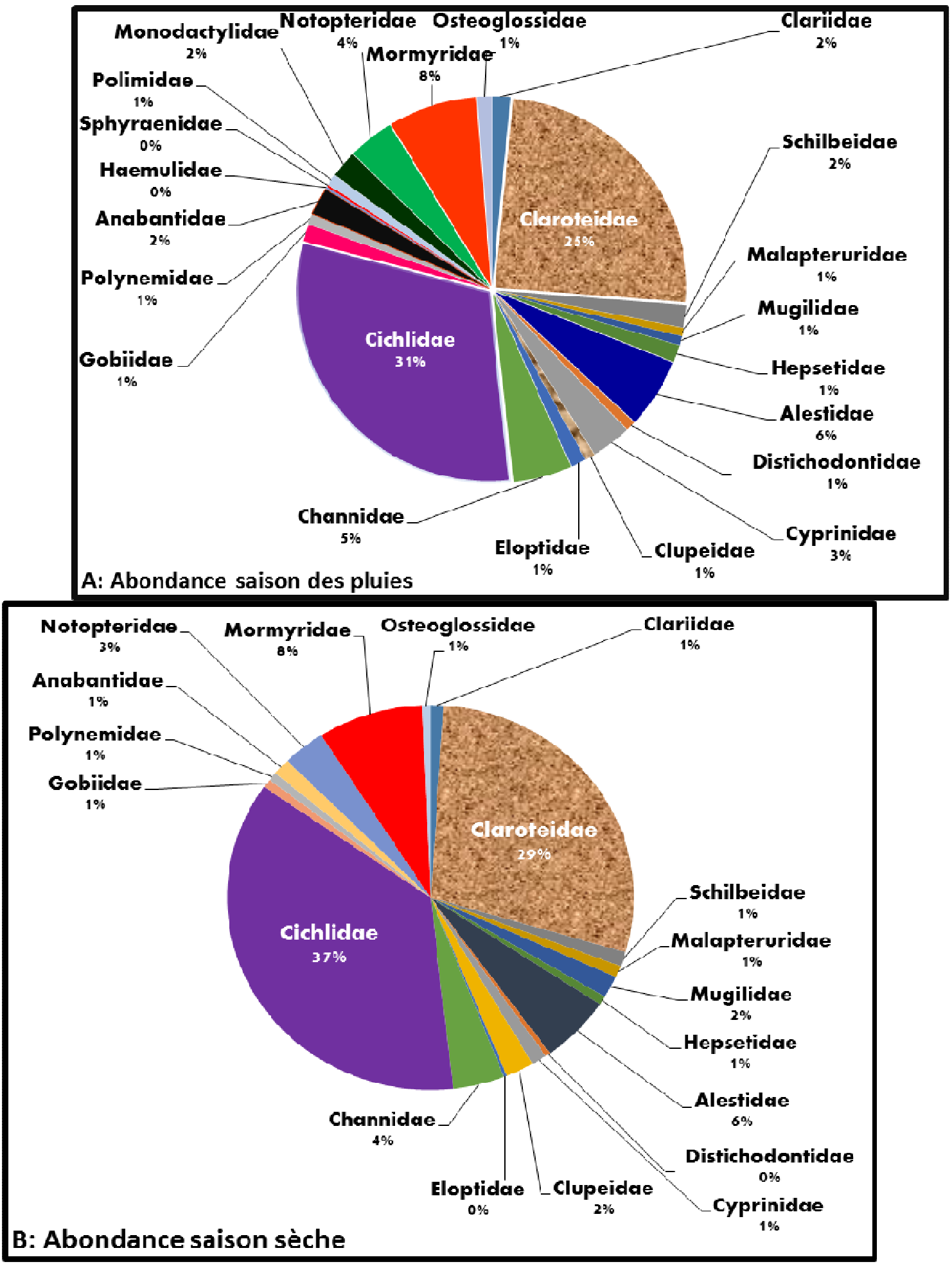

Figure 2 : Composition des captures par famille pendant les saisons des pluies $(A)$ et sèche $(B)$ dans la lagune Ono.

Indices de diversité spécifique de Shannon et d'équitabilité de Pielou: Les indices de diversité spécifique $\left(H^{\prime}\right)$ et d'équitabilité $(E)$ enregistrés ont été relativement élevés avec des valeurs respectives de 4,44 bits / individus et 0,85 (Tableau 3).Au niveau saisonnier, les indices de diversité spécifique de 


\section{Eyi et al. J. Appl. Biosci. 2016 Étude préliminaire des communautés ichtyofauniques de la lagune Ono (Côte d'Ivoire).}

Shannon et d'équitabilité ne présentent aucune variation significative (test de Mann-Whitney : $p>0,05$ ). En saison des pluies, l'indice de diversité de Shannon obtenu est de 4,57 pour un indice d'équitabilité de 0,87. En saison sèche, les indices de Shannon et d'équitabilité sont respectivement de 4,15 et de 0,82 .

Tableau 3 : Indice de diversité de Shannon et de Pielou (1966) calculé pour la faune ichtyologique de la lagune Ono de septembre 2015 à février 2016.

\begin{tabular}{l|c|c|c}
\hline Indices & Toute saison & Saison des pluies & Saison sèche \\
\hline Diversité spécifique $\left(\mathrm{H}^{\prime}\right)$ & 4,44 & 4,57 & 4,15 \\
\hline Diversité spécifique max $\left(\mathrm{H}_{\max }\right)$ & 5,22 & 5,25 & 5,06 \\
\hline Equitabilité $(\mathrm{E})$ & 0,85 & 0,87 & 0,82 \\
\hline
\end{tabular}

\section{DISCUSSION}

L'étude de la composition et de la structure de la diversité du peuplement ichtyologique de la lagune Ono a permis de récolter 39 espèces réparties en 28 genres, 23 familles et 8 ordres. Cette lagune comporte donc une faune ichtyologique assez diversifiée malgré sa petite taille. La comparaison avec la situation antérieure est difficile car il n'existe pas, dans la littérature, d'information sur la faune ichtyologique de cette lagune. L'importante richesse spécifique de la lagune Ono s'expliquerait par une diversité d'habitats au regard des indices de diversification et de l'adaptation de certaines espèces aux conditions du milieu (importante eutrophisation et faibles valeurs des paramètres physico-chimiques). En effet, les macrophytes aquatiques qui occupent plus de $50 \%$ de ce plan d'eau constituent une mosaïque de biotopes particuliers susceptible d'accueillir et d'abriter les différents peuplements ichtyologiques. Parmi ces macrophytes, l'on observe la forte présence d'une végétation herbeuse graminéenne flottante dominée par Echinochloa pyramidalis dont les caractéristiques écologiques favorisent une importante colonisation par les poissons. L'importante richesse de la faune ichtyologique de la lagune Ono s'explique aussi par le fait que cette lagune est en communication avec le fleuve Comoé qui comporte 55 espèces (Daget, 1960), d'où beaucoup d'espèces peuvent migrer dans la lagune. En outre, les faibles valeurs des paramètres environnementaux en général, et du $\mathrm{pH}$, de l'oxygène dissous et de la conductivité en particulier de cette lagune suggèrent l'inexistence de vie aquatique. Les valeurs élevées de l'oxygène dissous en octobre $(5,42 \pm 0,38 \mathrm{mg} / \mathrm{L})$ sont dues à la pluie qui a agité les eaux de surface juste avant les mesures, augmentant ainsi les échanges eau-atmosphère. Sinon, les valeurs de l'oxygène enregistrées au cours de la période d'étude n'ont pas excédé pas $2,5 \mathrm{mg} / \mathrm{L}$. Pourtant l'oxygène dissous est essentiel pour la respiration des organismes aquatiques et a donc une influence directe sur le fonctionnement biologique des milieux aquatiques. Selon Lévêque et Paugy (1999), les communautés ichtyologiques des cours d'eau africains sont très riches car beaucoup d'espèces s'adaptent aux conditions défavorables, notamment à la contraction de leur habitat durant la saison sèche.

Les Cichlidae, les Claroteidae, les Mormyridae et les Alestidae sont les familles les plus importantes en termes de nombre d'espèces, cet ordre semble ne pas être maintenu dans tous les cours d'eau. En effet, les travaux de Kantoussan, (2007) dans la rivière Baoulé du bassin du fleuve Sénégal au Mali, ont monté que ce sont les Cyprinidae suivies des Characidae et des Mormyridae puis des Mochokidae qui sont les plus représentées. Selon le même auteur, dans les barrages de Sankarani dans le lac de Sélingué et Manantali au Mali, ce sont plutôt les Cichlidae suivis des Mochokidae, des Mormyridae et des Alestidae qui sont les plus représentées. En termes de richesse spécifique les résultats de notre étude sont similaires aux travaux de Kuela, (2002) obtenus sur la Comoé dans sa partie burkinabè avec 40 espèces. Par contre, cette richesse spécifique est moins importante que celles d'autres cours d'eau ivoiriens et/ou africains comme la Comoé dans son ensemble où on dénombre 55 espèces (Daget, 1960) et le bassin du fleuve Niger avec 243 espèces.

Au niveau spécifique, les captures sont dominées en termes d'abondance et de biomasse par les espèces dulçaquicoles $(n=31)$ représentant $79,49 \%$ contre 06 espèces à affinité marine et/ou estuarienne (15,38\%) et 02 espèces introduites $(5,13 \%)$ dans les hydrosystèmes de la Côte d'Ivoire. Ces résultats sont en concordance avec ceux de plusieurs milieux d'eau douces (Kuela, 2002; Kantoussan, 2007; Konan, 2008). D'ailleurs, tenant compte de son fonctionnement hydrodynamique et ses caractéristiques géochimiques, Guiral et Ferhi (1989) ont qualifié cette lagune de réservoir d'eau douce, alimentée essentiellement par la 

(Côte d'Ivoire).

nappe phréatique. Cela justifie donc la présence de ce nombre élevé d'espèces dulçaquicoles. La présence d'espèces à affinité marine et/ou estuarienne est due à la proximité de la lagune Ebrié favorisant ainsi la remontée des taxons provenant de ce milieu halin qui ont besoin de remonter les cours d'eau à un moment de leur cycle de vie. En effet, Lévêque et Paugy (1999) et De Mérona (2005) font remarquer que les espèces migratrices en provenance des milieux saumâtres remontent vers l'amont des fleuves et rivières au moment des crues pour la ponte. La communication permanente entre lagune Ebrié-Comoé favorise la présence régulière de telles espèces dans la lagune Ono toute l'année. En outre, une espèce de tilapia ayant les traits à la fois de Tilapia guineensis et de Tilapia zilli a été fortement rencontrée dans les

\section{CONCLUSION}

La présente étude a permis d'entamer l'étude préliminaire relative à la faune ichtyologique de la lagune Ono. Trente-neuf espèces, dont une espèce de tilapia hybride, ont été signalées pour la première fois. La lagune Ono pourrait subir une importante eutrophisation due à la forte anthropisation de ses berges et son bassin. Par conséquent, un suivi

\section{REMERCIEMENTS}

Cette étude s'inscrit dans la série des travaux monographiques réalisée au Centre de Recherches Océanologiques d'Abidjan sur la biodiversité et la bioécologie des espèces à potentialités aquacoles exploitées par la pêche lagunaire ivoirienne. Les

\section{REFERENCES BIBLIOGRAPHIQUES}

Albaret J.J., 1994. Les poissons, biologie et peuplements. In: Durand J-R., Dufour P., Guiral D. \& Zabi S.G.F., Eds. Environnement et ressources aquatiques de Côte-d'Ivoire (Tome II). Paris, France : ORSTOM. pp. 239279.

Brummett R.E., Lazard J. \& Moehl J., 2008. African aquaculture: Realizing the potential. Food Policy 33: 371-385.

Daget J. \& Iltis A., 1965. Poissons de Côte d'Ivoire (eaux douces et saumâtres). Edition IFAN DAKAR, 385p

Daget J., 1960. Les poissons de la Volta Noire et de la Haute Comoé. Bulletin du Muséum National d'Histoire Naturelle Paris, (2) 32: 320-330.

Dajoz R., 2000. Précis d'écologie. 7ème Edition. Dunod, Paris, 615p. captures. Les caractéristiques de ce poisson qui a colonisé la plupart des lagunes de Côte d'Ivoire ont déjà été décrites par Nobah et al. (2008). En toute saison confondue, les indices $H^{\prime}$ et $E$ sont respectivement de 4,44 et 0,85 . Ces indices sont respectivement de 4,57 et 0,87 en saison des pluies et de 4,15 et 0,82 en saison sèche. Les fortes valeurs indicielles enregistrées sont caractéristiques des communautés diversifiées avec un fort degré d'organisation. Cependant, ces deux indices restent dépendants de la taille des échantillons et du type d'habitat. II est donc souhaitable de combiner ces indices synthétiques à des méthodes complémentaires telles que les analyses multi-variées et surtout de ne pas tirer des conclusions qui vont au-delà de ce que permettent ces indices.

permanent de l'état de la faune ichtyologique s'avère nécessaire dans l'élaboration des mesures adéquates à prendre pour éviter une perte de la biodiversité. Les résultats de ces investigations serviront de données de références de l'ichtyofaune de la lagune Ono et enrichiront le répertoire national de la faune ichtyologique de la Côte d'Ivoire.

auteurs tiennent à remercier les autorités villageoises et coutumières de la localité de Ono-Salci et l'ensemble des pêcheurs qui ont contribué à la réalisation de cette étude.

De Mérona B., 2005. Le fleuve, le barrage et les poissons : Le Sinnamary et le barrage de Petit-Saut en Guyane française. Éditions IRD, Paris, 135p.

Diversité ichtyologique d'un bassin ouest-africain après la construction d'un barrage. Cybium 23 (2): 147-160.

Ettien N. \& Guiral D., 1991. Les Macrophytes Aquatiques des berges lagunaires. Journal Ivoirien d'Océanologie et de Limnologie, 1 (2) : 24-40.

Gourène G., Teugels G.G., Hugueny B. \& Thys van den Audenaerde D.F.E., 1999. Évaluation de la diversité ichtyologique d'un bassin ouest africain après la construction d'un barrage. Cybium 23(2): 147-160. 
Guiral D., Albaret J.- J., Baran E., Bertrand F., Debeney J.-P., Diouf P.-S., Guillou J.-J., Le Loeuff P.,

Montoroi J.-P., Sow M., 1999, "Les écosystèmes à mangrove », dans Rivières du sud : sociétés et mangroves ouest-africaines, éd. Par M.- C. Cormier-Salem, Paris, I.R.D, p. 63-130.

Guiral D \& Ferhi A., 1989. Caractérisation ionique et isotonique d'un système hydrologique tropical: la lagune Ebrié (Côte d'lvoire). Oceanologica Acta, 12(1) : 47-55.

Kantoussan J., 2007. Impacts de la pression de pêche sur l'organisation des peuplements de poissons : application aux retenues artificielles de Sélingué et de Manantali, Mali, Afrique de I'Ouest. Thèse de doctorat, Agrocampus Rennes, $195 \mathrm{p}$.

Konan K.F., 2008. Composition, structure et déterminisme de la diversité ichtyologique des rivières côtières du Sud-est de la Côte d'Ivoire (Soumié-Eholié-Ehania-Noé). Thèse de doctorat, Université d'Abobo-Adjamé, 146.

Kuela J.M.D., 2002. Étude des peuplements ichtyologiques de la Comoé et des modes d'exploitation piscicole dans la zone agrosylvo-pastorale du projet GEPRENAF. Mémoire de fin d'étude, Université. Polytechnique de Bobo Dioulasso, 73 pages.

Lévêque C., Paugy D \& Teugels G.G. (Eds.) 1990. Faune des poissons d'eaux douces et saumâtres de l'Afrique de l'ouest. Tome I. Faune tropicale, XXVIII, MRAC-Tervuren. ORSTOM, Paris : 1-384.

Lévêque C. 1994. Introduction générale : Biodiversité des poissons africains. In: Diversité biologique des poissons des eaux douces et saumâtres d'Afrique (Teugels G. G., Guégan J. -F. \& Albaret J. -J., eds). Annales du Musée Royal de l'Afrique Centrale 275: 7-16.

Lévêque C. \& Paugy D., 1999. Impacts des activités humaines. In: Les Poissons des eaux continentales africaines. Diversité, Écologie, Utilisation par l'homme. Edition IRD, Paris : 365-383.

Lévêque C. \& Paugy D., 2006. Distribution géographique et affinités des poissons d'eau douce africains pp. 59-74, in: C. Lévêque et D. Paugy (éditeurs), Les poissons des eaux continentales. Diversité, écologie, utilisation par l'homme, Éditions IRD, Paris, $521 \mathrm{p}$.

Paugy D., Lévêque C \& Teugels G.G., 2003. Faune des poissons d'eaux douces et saumâtres de
I'Afrique de l'Ouest. Tome 1. IRD Éditions, MNHN, Paris, France \& MRAC, Tervuren, Belgique. 457 pp.

Pielou E.C., 1975. Ecological diversity. John Wiley \& Sons Inc. 176p.

Pielou E. C., 1966. The measurement of diversity in different types of biological collections. , Journal of Theoretical Biology, 13, 131-44.

Nobah C.S.K., Tidiani K., Ouattara I.N., Kouamelan P.E., N'douba V. \& Snoeks J., 2008. Étude des performances de croissance de deux tilapias (Tilapia zillii et $T$. guineensis) et de leurs hybrides en cage flttante Cybium 32(2): 131-136

Teugels G.G., Lévêque C., Paugy D. \& Traoré K., 1988. État des connaissances sur la faune ichtyologique des bassins côtiers de la Côte d'lvoire et de l'Ouest du Ghana. Revue d'Hydrobiologie tropicale, 21 (3):221- 237. 